\title{
Detección, evaluación y pronóstico de las sequías en la región del Organismo de Cuenca Pacífico Norte, México
}

\author{
Ravelo, A.C.; R. Sanz Ramos y J.C. Douriet Cárdenas
}

\begin{abstract}
RESUMEN
Las cuencas hidrológicas en la región del Organismo de Cuenca Pacífico Norte en México son afectadas por recurrentes sequías que impactan negativamente a la agricultura y a todas las actividades socio-económicas en general. Para el estudio de las sequías se consideró el período 1978-2012. Se utilizaron datos de precipitación, evapotranspiración potencial, satelitales de MODIS y capacidad edáfica de retención hídrica de 54 estaciones meteorológicas de la región. Se calcularon los siguientes índices: estandarizado de precipitación, de severidad de sequía de Palmer y de diferencia normalizada de vegetación. Estos índices permitieron la detección, la evaluación y el pronóstico de la intensidad de las deficiencias hídricas en períodos con sequías o sin éstas. El pronóstico de las sequías fue realizado con redes neuronales y validado estadísticamente. La cartografía de los índices fue automatizada mediante el desarrollo de un programa de computación específico (SOS). Se consideraron dos períodos con condiciones hídricas extremas: 2004-2005, cuando los índices señalaron condiciones de exceso hídrico, y 2011-2012 cuando se presentaron sequías severas que afectaron las reservas de las represas, los caudales de los ríos y la disponibilidad de agua en zonas urbanas y agrícolas. Estas condiciones fueron verificadas con informes oficiales del gobierno y periodísticos.
\end{abstract}

Palabras clave: índices, sequía, redes neuronales, México

Ravelo, A.C.; R. Sanz Ramos and J.C. Douriet Cárdenas, 2014. Detecting, assessing and forecasting droughts in the region of the North Pacific Watershed Organism, Mexico. Agriscientia 31 (1): 11-24

\section{SUMMARY}

The watersheds in the region of the North Pacific Watershed Organism in Mexico are affected by recurring droughts which impact negatively the agriculture and every socio-economic activity. The drought study considered the period 1978-2012 and used data of rainfall, potential evapotranspiration, MODIS satellite and soil water holding capacity for 54 weather stations of the region. Several drought indices were computed: Standard precipitation index, Palmer drought severity index, and the normalized difference vegetation index. These indices allowed the detection, assessment and forecasting of intensity 
of the hydrological deficits during drought or not drought periods. A neural network was used for the drought forecasting and statistically validated. The mapping of indices was done automatically with a computer program (SOS). Two periods with opposite extreme hydrological conditions were considered: 2004-2005 when the indices indicated water excesses and 2011-2012 when severe droughts affected the water reservoirs, river flows and water availability in urban and rural areas. These conditions were verified with government and newspaper reports.

Key words: índices, drought, neural network, México

A.C. Ravelo: CREAN / CONICET. Universidad Nacional de Córdoba. Ciudad Universitaria. Av. Valparaíso s/n, Córdoba, Argentina. R. Sanz Ramos y J.C. Douriet Cárdenas: CONAGUA / OCPN. Culiacán, Sinaloa, México. Correspondencia a: ravelo43@gmail.com

\section{INTRODUCCIÓN}

La sequía es un fenómeno natural que se origina por deficiencias en la precipitación durante un tiempo extendido, y es una anomalía temporal que puede suceder en casi todas las zonas climáticas, aun en aquellas que tengan normalmente elevadas precipitaciones (Morales et al., 2000). Las sequías se distinguen de la aridez, dado que ésta corresponde a regiones que poseen normalmente escasas precipitaciones y es una característica permanente del clima (Ravelo, 2000).

Las sequías pueden clasificarse (Ravelo, 2012) según su génesis y/o efectos adversos, en:

1) Sequía meteorológica: se presenta cuando la precipitación es muy inferior a la esperada y la deficiencia hídrica se extiende por un largo período. La intensificación y prolongación en el tiempo de esta clase conduce a la aparición de los otros tipos de sequía.

2) Sequía agrícola: se registra cuando la cantidad de precipitación y su distribución, las reservas de agua del suelo y las pérdidas debidas a la evaporación se combinan, para causar una disminución considerable de los rendimientos de cultivos y la productividad del ganado por deterioro de las pasturas.

3) Sequía hidrológica: ocurre cuando hay un déficit continuo en la escorrentía de superficie y alcanza ésta un nivel inferior a las condiciones normales y/o cuando disminuye el nivel de las aguas subterráneas.

4) Sequía socio-económica: es consecuencia de la ocurrencia de los tipos de sequías anteriores, por lo cual la comunidad y sus actividades económicas se ven severamente afectadas.
Las clases enunciadas se presentan en el orden de aparición del evento y con el consecuente impacto negativo.

Los comienzos de una sequía son poco perceptibles, pero una vez establecida puede identificarse por sus efectos negativos sobre la producción de cultivos, las condiciones de pastoreo, el riesgo de incendios — debido a la acumulación de materias secas- y la aceleración de la desertificación por la pérdida de cobertura vegetal en terrenos fácilmente erosionables. Todo esto trae aparejado fuertes pérdidas en actividades agrícolas, ganaderas e industriales y también consecuencias sociales y económicas conexas, como son la inseguridad en los suministros alimentarios, y pérdidas económicas en el sector agrícola ganadero e industrial (Ravelo, 1980; Planchuelo \& Ravelo, 1985; White \& Walcott, 2009). Las pérdidas económicas son similares y en algunos casos muy superiores a las ocurridas con otras catástrofes naturales (huracanes, inundaciones, incendios, etc.).

Considerando las cuantiosas pérdidas económicas y sus repercusiones en la sociedad, la Convención para la Lucha contra la Desertificación y las Sequías de la Organización de la Naciones Unidas (ONU) ha centralizado los esfuerzos internacionales para establecer estrategias de respuesta y mitigación a las sequías (EIRD/ONU, 2009). En Estados Unidos se ha conformado una comisión que ha definido los lineamientos para que las instituciones de los sectores públicos y privados adopten medidas que ayuden a mitigar los efectos de las sequías (USNDPC, 2000).

En el caso particular de México, las prolongadas y severas sequías ocurridas en diferentes y extensas partes del país ha determinado acciones en los distintos niveles gubernamentales, con la finali- 
dad de crear planes y programas de gestión para contrarrestar sus efectos mediante la prevención y la mitigación (CONAGUA, 2014). Asimismo, se han realizado estudios a nivel regional en el estado de Sinaloa para caracterizar y evaluar el efecto de las sequías (Douriet, 2010; Sanz Ramos et al., 2012). En la región del Organismo de Cuenca Pacífico Norte (OCPN/CONAGUA), la presencia de sequías recurrentes en diferentes fases de severidad, intensidad, duración y extensión, ha provocado importantes impactos negativos en los sectores ambiental, económico y social (Ravelo, 2012). En épocas recientes se han presentado sequías extremas de gran magnitud y extensión, como las ocurridas en el periodo 1998-2004 y la de 2011-2012. Esta última es considerada como la más severa de las últimas décadas y es responsable de grandes pérdidas en la agricultura, ganadería y economías locales. También se observaron escurrimientos superficiales prácticamente nulos y presas vacías, compensado en parte por una extracción intensiva de agua subterránea en los acuíferos costeros de la región, lo que afectó sus niveles estáticos (CONAGUA, 2012). Además, se aumentó el riesgo de la sobreexplotación acuífera permanente y el fenómeno de la intrusión salina; esto generó una tensión social en la disputa por el agua disponible, manifestado por robos de agua en canales y una gran cantidad de perforaciones de pozos clandestinos (Douriet, 2013a).

Si bien no existen mecanismos que puedan evitar la ocurrencia de sequías, es posible, mediante el análisis de la información climática, meteorológica e hidrológica, realizar una identificación, valoración y previsión del fenómeno ambiental. Estas acciones pueden permitir preparar planes de prevención de daños o de mitigación de los efectos negativos de las sequías (Zanvettor \& Ravelo, 2000; Ravelo et al., 2001). Algunos elementos de análisis consisten en índices de sequía de tipo meteorológico; por ejemplo, el índice de severidad de sequía (PDSI, siglas de Palmer drought severity index) de Palmer (1965) y el índice estandarizado de precipitación (SPI, siglas de standarized precipitation index) de McKee et al. (1993). El índice de diferencia normalizada de vegetación (NDVI, siglas de normalized difference vegetation index) es derivado de información satelital y puede ser utilizado para el monitoreo y evaluación de las sequías (Kogan, 1997; Peters et al., 2002). Sus ventajas son la amplia cobertura geográfica, su complementación con otros índices meteorológicos y su relación con la productividad de los cultivos.

El índice de severidad de sequía (PDSI) se continúa utilizando en numerosos países como un indicador confiable de las condiciones hídricas, a pesar de las limitaciones destacadas por Alley (1984). En la Argentina (CREAN, 2012) y en Estados Unidos (NOAA, 2012) se publican periódicamente los índices PDSI y SPI, los cuales permiten identificar condiciones hídricas extremas y su evolución en el tiempo. Ravelo et al. (2012) presentaron el desarrollo de un atlas de sequías en la Argentina para el período 1980-2011 utilizando los índices PDSI, SPI y NDVI. En Estados Unidos, Hayes et al. (1999) identificaron correctamente las sequías de 1996 utilizando el SPI, mientras que en la Argentina Zanvettor \& Ravelo (2000) utilizaron el mismo índice para evaluar la sequía de 1999-2000.

Los índices resumen en una o varias variables de relevancia el proceso de las sequias y pueden ser graficados de forma de visualizar su variabilidad temporal o geográfica, además de su utilización en métodos de pronóstico (Mishra \& Desai, 2006) y de evaluación de la vulnerabilidad y riesgo de sequías (Ravelo, 2012).

El objetivo de este trabajo fue desarrollar y aplicar un sistema de detección, evaluación y pronóstico de las condiciones hídricas ambientales, especialmente de sequías, empleando índices de reconocida eficiencia y complementariedad, para ser utilizado en la planificación de acciones de alerta temprana y mitigación por parte de los sectores público y privado de la sociedad.

\section{MATERIALES Y MÉTODOS}

\section{Zona de estudio}

La zona de estudio comprende a las cuencas del Organismo de Cuenca Pacífico Norte (OCPN) perteneciente a la Comisión Nacional de Agua (CONAGUA), México. Dichas cuencas se encuentran ubicadas en los estados de Sinaloa, Sonora, Chihuahua, Durango, Nayarit y Zacatecas (Figura 1) y comprenden un área de $156.627 \mathrm{~km}^{2}$. Los principales ríos son: Fuerte, Sinaloa, Mocorito, Tamazula, Humaya, Culiacán, San Lorenzo, Elota, Piaxtla, Quelite, Presidio, Baluarte, Cañas, Acaponeta y San Pedro; sus mayores presas son Luis Donaldo Colosio (Huites), Miguel Hidalgo (El Mahone), Gustavo Diaz Ordaz (Bacurato), Eustaquio Buelna, Adolfo Lopez Mateos (El Varejonal), Sanalona, Jose Lopez Portillo (Comedero), Aurelio Benassini (El Salto) y Picachos. Dichas presas permiten, además de proveer agua para consumo urbano e industrial, la irrigación de 805.200 ha en las que los cultivos más importantes son maíz, frijol, trigo, tomate, caña de azúcar, alfalfa, forrajeras, árboles frutales y legumbres.

La información geográfica sobre las estaciones 


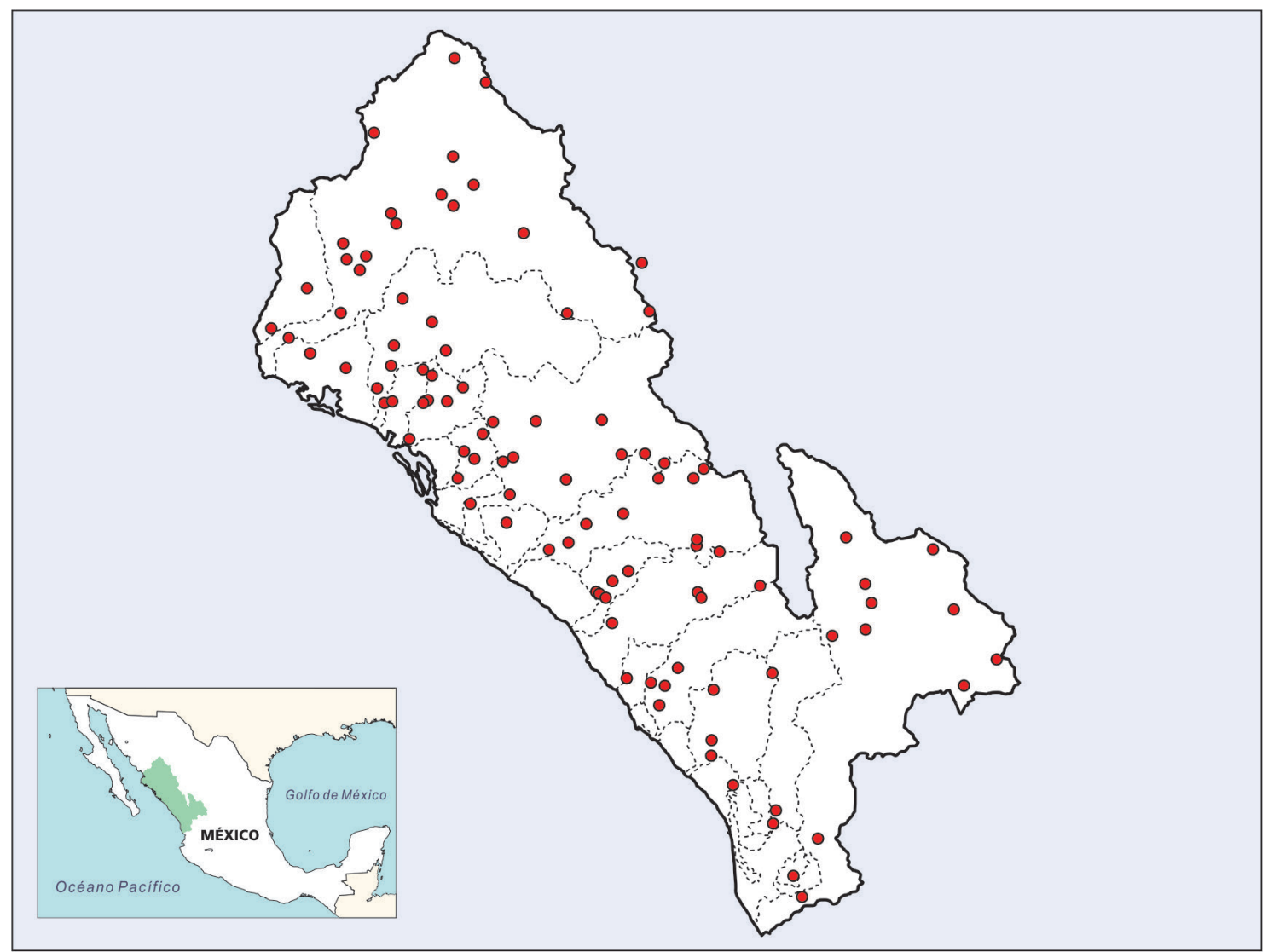

Figura 1. Área de estudio en el Organismo de Cuenca del Pacífico Norte que comprende áreas de los estados de Sinaloa, Sonora, Chihuahua, Durango y Zacatecas. Los puntos rojos indican las estaciones meteorológicas utilizadas en el estudio.

seleccionadas y las capas de información correspondiente a los municipios, trayectoria de los ríos y delimitación del área geográfica en estudio, fue obtenida de la base de datos de CONAGUA. Esta información fue convertida a formato IDRISI (Eastman, 2012), para generar las máscaras y capas de presentación final de los mapas de sequía para toda la región. La interpolación espacial se realizó mediante el método krigging del programa IDRISI.

\section{Materiales}

Se utilizaron series temporales de datos mensuales de precipitación y evapotranspiración potencial y las capacidades de retención hídrica de los suelos. Estas capacidades fueron estimadas a partir de las características texturales del suelo y luego utilizadas para el cálculo del balance hídrico del índice de Palmer (1965).

Se obtuvieron series temporalmente concomitantes de 1978 a 2012 para valores diarios de precipitación de 54 estaciones meteorológicas situadas en las cuencas (CONAGUA, 2012). La evapotranspiración potencial promedio para cada mes (en $\mathrm{mm} /$ día) se obtuvo de IWMI (2012). En el caso de las estaciones que no disponían de datos se procedió a estimar la evapotranspiración por el método del gradiente altimétrico (Ravelo, 1973).

Se utilizaron imágenes mensuales del NDVI derivadas de datos logrados por el satélite Terra (sensor MODIS) para el período 2000-2012 (Justice \& Townshend, 2002).

Los datos meteorológicos fueron sometidos a un estricto control de calidad en lo referente a continuidad, variabilidad y magnitud. La continuidad fue establecida de forma de no utilizar series incompletas por datos faltantes, lo cual determinó la eliminación del análisis de varias estaciones. La variabilidad de la serie y la magnitud de los datos fueron controlados estadísticamente. Se identificaron a aquellas series y/o datos sospechosos cuyos valores se encontraban fuera del rango establecido por la suma y la resta de tres desviaciones estándar al promedio. Considerando tres desviaciones 
por encima o debajo del valor medio se pudieron identificar valores incorrectos con una probabilidad de ocurrencia igual o inferior al $1 \%$, y se complementó la verificación de la validez del dato o la serie mediante un análisis comparativo de los registros meteorológicos de las estaciones vecinas.

Los datos de reservas hídricas de las presas del OCPN fueron provistos por CONAGUA (2012).

\section{Métodos}

Se utilizaron los siguientes índices de sequía: índice estandarizado de precipitación (SPI) para 12 meses (McKee et al., 1993), índice severidad de sequía de Palmer (PDSI) (Palmer, 1965; Ravelo \& Herrero, 1999) e índice de vegetación de diferencia normalizada (NDVI) (Tucker, 1979; Liu and Kogan, 1996; Kogan, 1997). Estos índices permitieron el monitoreo temporal y la evaluación de la intensidad de la sequía.

El pronóstico de las sequías fue realizado con el uso de redes neuronales (Mishra \& Desai, 2006) y los índices PDSI y SPI mensuales. Las condiciones futuras de sequías para los próximos 3 meses se realizó para estaciones selectas y se graficaron los resultados. La precisión de los pronósticos del PDSI para 1, 2 y 3 meses fue determinada mediante el uso del coeficiente de Pearson y de los errores medios absolutos y cuadráticos medios. Se consideraron las estaciones Culiacán, Varejonal y SanaIona, que contaban con datos para los años 2011, 2012 y 2013, y se analizaron estadísticamente 247 valores pronosticados.

La detección y evaluación de las sequías se realizó mediante el uso de diferencias de imágenes del NDVI. La diferencia de imágenes (NDVIDif) se generó al restar una imagen de un mes determinado, en el cual los índices de sequía indicaban la ocurrencia del evento, y una imagen promedio (2000-2012). La magnitud de las diferencias negativas indica la intensidad de la sequía, mientras que diferencias positivas indican una situación de abundancia hídrica.

El cálculo de los índices y su representación gráfica se realizó mediante el programa de computadora denominado Sistema Operativo de Sequias (SOS), diseñado conceptualmente por Ravelo et al. (2001). EI SOS consiste en un programa desarrollado en lenguaje $\mathrm{C}++$ y utiliza módulos del sistema operativo Windows e IDRISI para su ejecución y obtención de resultados numéricos y cartográficos (Ravelo, 2012).

La validación de la capacidad del sistema para identificar extremos hídricos (sequías y excesos hídricos) y, específicamente, para monitorear, evaluar y pronosticar la intensidad y distribución geográfica de las sequías, fue realizada para un período con exceso de humedad (2004-2005) y para un período con sequías (2011-2012). La verificación de la ocurrencia y extensión de los extremos hídricos en ambos períodos se realizó considerando los informes oficiales de CONAGUA sobre el estado de almacenamiento de agua de las presas (CONAGUA, 2012). También se utilizaron las publicaciones de los municipios afectados por la sequía (CONAGUA, 2014).

\section{RESULTADOS Y DISCUSIÓN}

El análisis de la serie temporal del PDSI permitió identificar a los períodos 2004-2005 y 2011-2012 como extremos en las condiciones hídricas de exceso y deficiencia, respectivamente. La Figura 2 presenta una serie temporal del PDSI para la localidad de Guadalupe y Calvo (Chihuahua). En ella se observa un índice positivo superior a 4 durante un período de varios meses desde fines del 2004 a comienzos del 2005. Las condiciones correspondieron a humedad excesiva, y similares condiciones se registraron en otras localidades del OCPN, lo que determinó que dicho período se lo caracterizara como el más húmedo de los últimos 20 años.

El exceso de humedad se evidencia en la parte centro y norte de la región del OCPN, según lo indica el PDSI (Figura 3). EI PDSI señala condiciones de humedad incipiente a excesiva, excepto el sudeste de la región donde se observó humedad normal. Esta situación hídrica trajo aparejados una excelente recarga de los acuíferos y aumento de las reservas hídricas. En febrero de 2005 las presas presentaron adecuadas reservas (Tabla 1)

La Figura 4 presenta el SPI de febrero de 2005

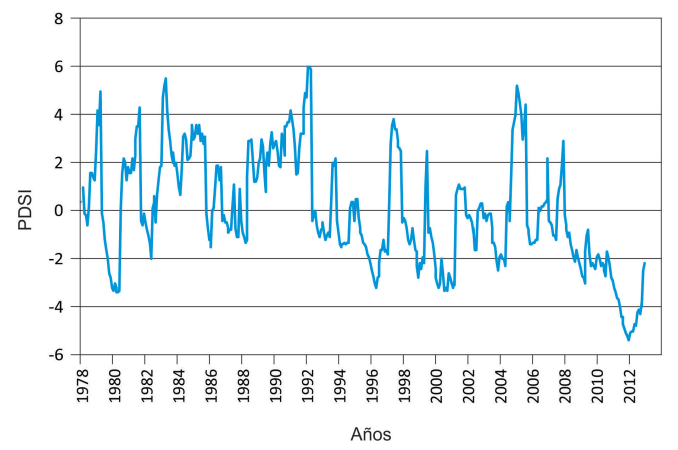

Figura 2. Variabilidad mensual del índice de sequía de Palmer (PDSI) para la localidad de Guadalupe y Calvo (Chihuahua) en el período 1978-2012. 


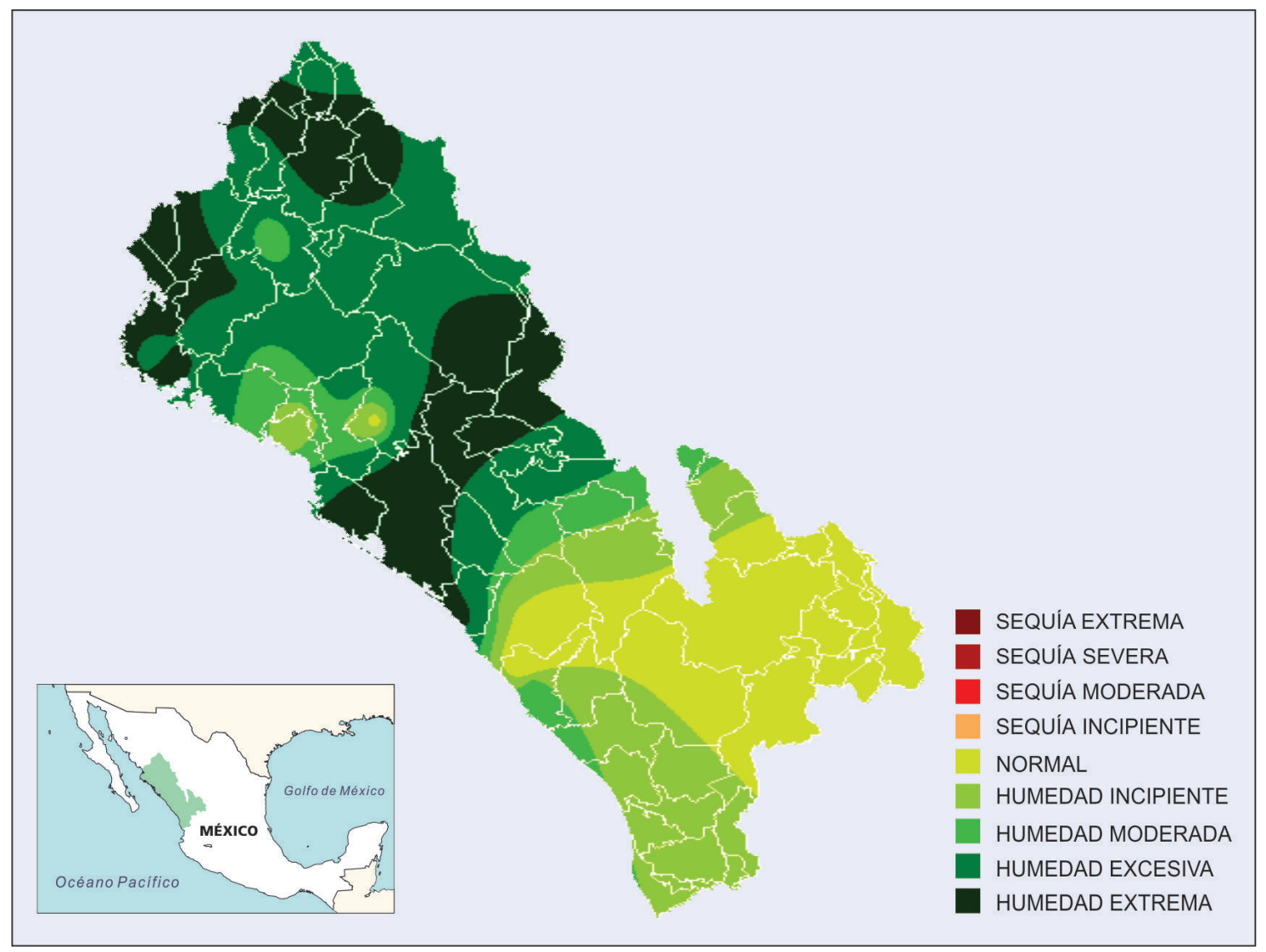

Figura 3. Índice de sequía de Palmer (PDSI) para febrero de 2005 en el área de estudio.

Tabla 1. Almacenamiento de las presas en el Organismo de Cuenca del Pacífico Norte durante los meses de febrero de 2005 y 2012.

\begin{tabular}{lccccc}
\hline & & \multicolumn{2}{c}{ Febrero 2005} & \multicolumn{2}{c}{ Febrero 2012 } \\
\cline { 3 - 6 } \multicolumn{1}{c}{ PRESAS } & $\begin{array}{c}\text { Capacidad total } \\
\left(\mathrm{Mm}^{3}\right)\end{array}$ & $\begin{array}{c}\text { Almacenamiento } \\
\left(\mathrm{Mm}^{3}\right)\end{array}$ & \% $\|_{\text {llenado }}$ & $\begin{array}{c}\text { Almacenamiento } \\
\left(\mathrm{Mm}^{3}\right)\end{array}$ & \% llenado \\
\hline Luis Donaldo Colosio & 4568,0 & 2803,06 & 61,4 & 716,11 & 15,7 \\
Miguel Hidalgo & 3917,1 & 1448,78 & 37,0 & 760,72 & 19,4 \\
Gustavo Diaz Ordaz & 2822,7 & 1771,59 & 62,8 & 287,69 & 10,2 \\
Eustaquio Buelna & 265,0 & 107,857 & 40,7 & 28,701 & 10,8 \\
Adolfo Lopez Mateos & 3086,6 & 2940,45 & 95,3 & 375,16 & 12,2 \\
Sanalona & 673,5 & 546,785 & 81,2 & 175,44 & 26,0 \\
Jose Lopez Portillo & 3966,2 & 1586,26 & 40,0 & 435,49 & 11,0 \\
Aurelio Benasini & 810,0 & 374,987 & 46,3 & 140,32 & 17,3 \\
\hline
\end{tabular}

calculado con la precipitación de los 12 meses previos. Se observan excesos hídricos en casi toda la región, excepto en dos áreas costeras restringidas. Este índice señala la abundante disponibilidad hídrica de los ríos y la consecuente recarga de las presas durante el período considerado. Estas condiciones han sido verificadas utilizando los registros de CONAGUA (2012).

La evaluación satelital de la situación hídrica obtenida mediante el NDVIDif se ilustra en la Figura 5. Toda la región presenta valores del NDVI por encima del valor promedio, lo cual es un indicador de una vegetación vigorosa que resulta de la abundante disponibilidad hídrica de los meses anteriores.

En el otro extremo de condiciones hídricas se encuentra el período 2011-2012, que presentó sequías generalizadas en extensas regiones del país, 


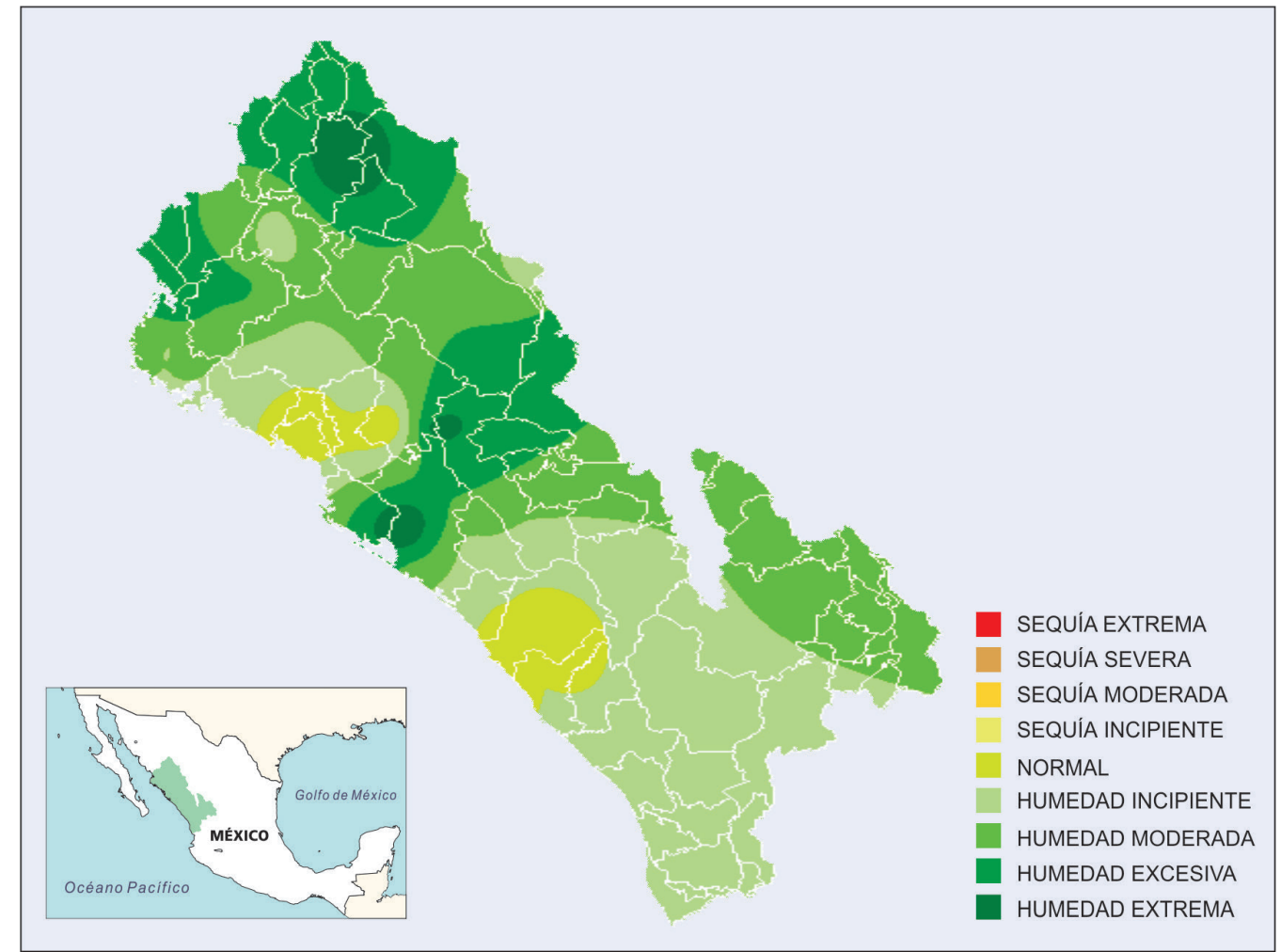

Figura 4. Índice estandarizado de precipitación SPI12 para febrero de 2005 en el área de estudio.

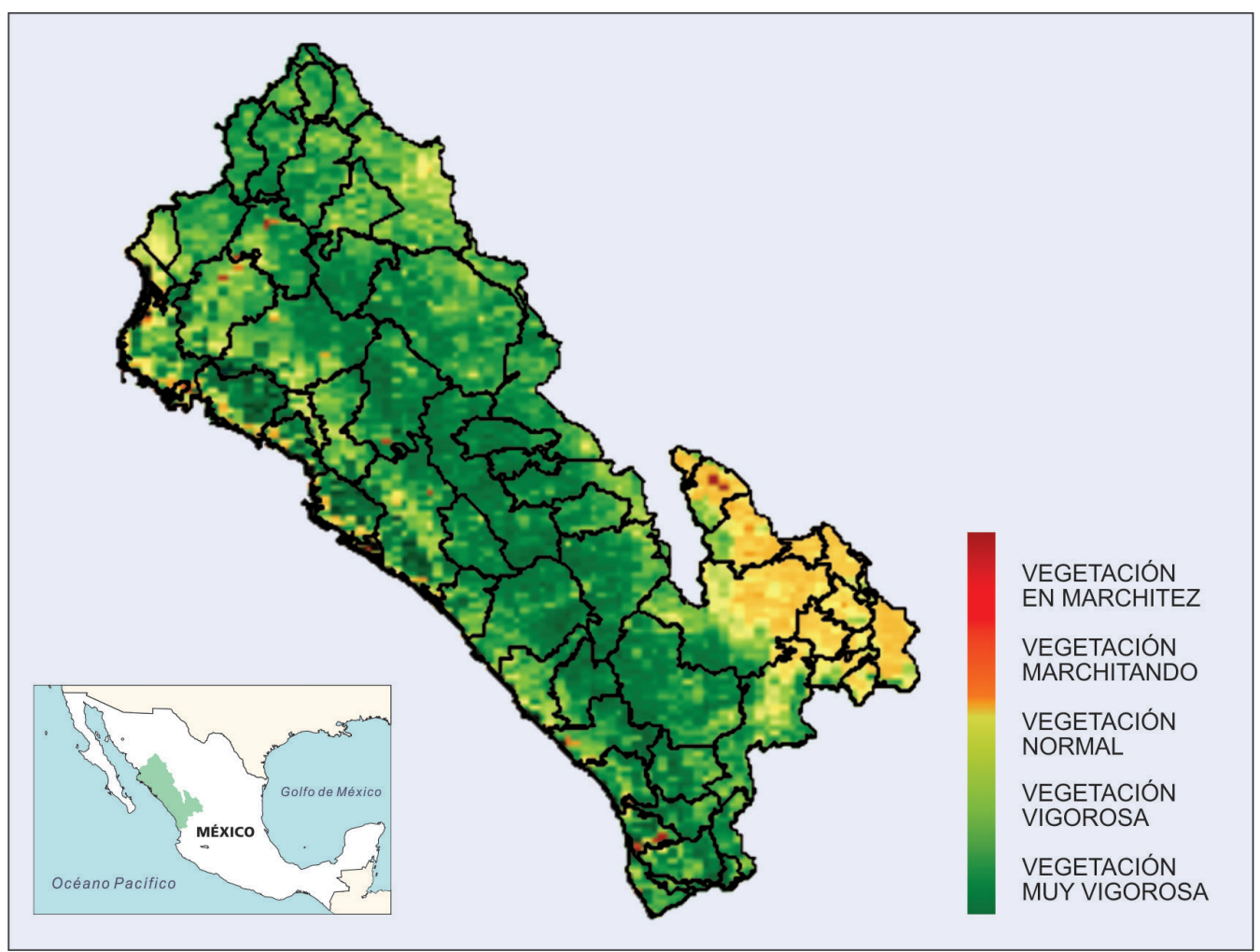

Figura 5. Índice de vegetación (NDVIDif) para febrero de 2005 en el área de estudio. 
incluyendo la correspondiente al OCPN. Los valores del PDSI oscilaron entre -2,2 y -5,1 lo que señala condiciones de sequías moderadas a extremas. La Figura 6 presenta el PDSI para febrero de 2012, donde se aprecian las sequías generalizadas en toda la región, excepto en áreas restringidas, generalmente costeras del centro y sur. Las cuencas medias y altas fueron las más afectadas.

El SPI de 12 meses señala que los cursos de agua (arroyos y ríos) en las partes medias y altas de las cuencas (Figura 7), hacen aportes hídricos escasos o nulos a las presas y estas presentaron ínfimas reservas para el mes de febrero de 2012 (Tabla 1).

EI NDVIDif identificó extensas áreas con valores por debajo del valor promedio, lo cual señaló la presencia de una vegetación con deficiencias hídricas. Sólo áreas muy circunscriptas presentaban condiciones normales, probablemente asociadas a agricultura con riego (Figura 8).

En 2012 los informes oficiales y periodísticos presentaban los impactos adversos de las severas y generalizadas deficiencias hídricas que afectaron las reservas de las represas (Tabla 1), los caudales de los ríos y la disponibilidad de agua en zonas urbanas y agrícolas. Dadas las condiciones mencionadas, las autoridades del OCPN debieron realizar una administración muy cuidadosa de los escasos recursos hídricos de forma de minimizar los efectos adversos de la sequía en las actividades agrícolas bajo riego. Las regiones sin riego se vieron muy afectadas en la producción de granos y pasturas.

En el caso de ocurrencia de sequias generalizadas y a los fines de una administración adecuada del agua disponible en las presas, es importante disponer de un pronóstico de las condiciones hídricas para los próximos meses. En la Figura 9 se presenta el pronóstico de la sequía realizado en febrero para los meses de marzo, abril y mayo de 2012 para la localidad de El Varejonal. La precisión del pronóstico para el mes siguiente fue realizada en enero y verificada en febrero. Puede apreciarse que existe una pequeña diferencia entre el valor

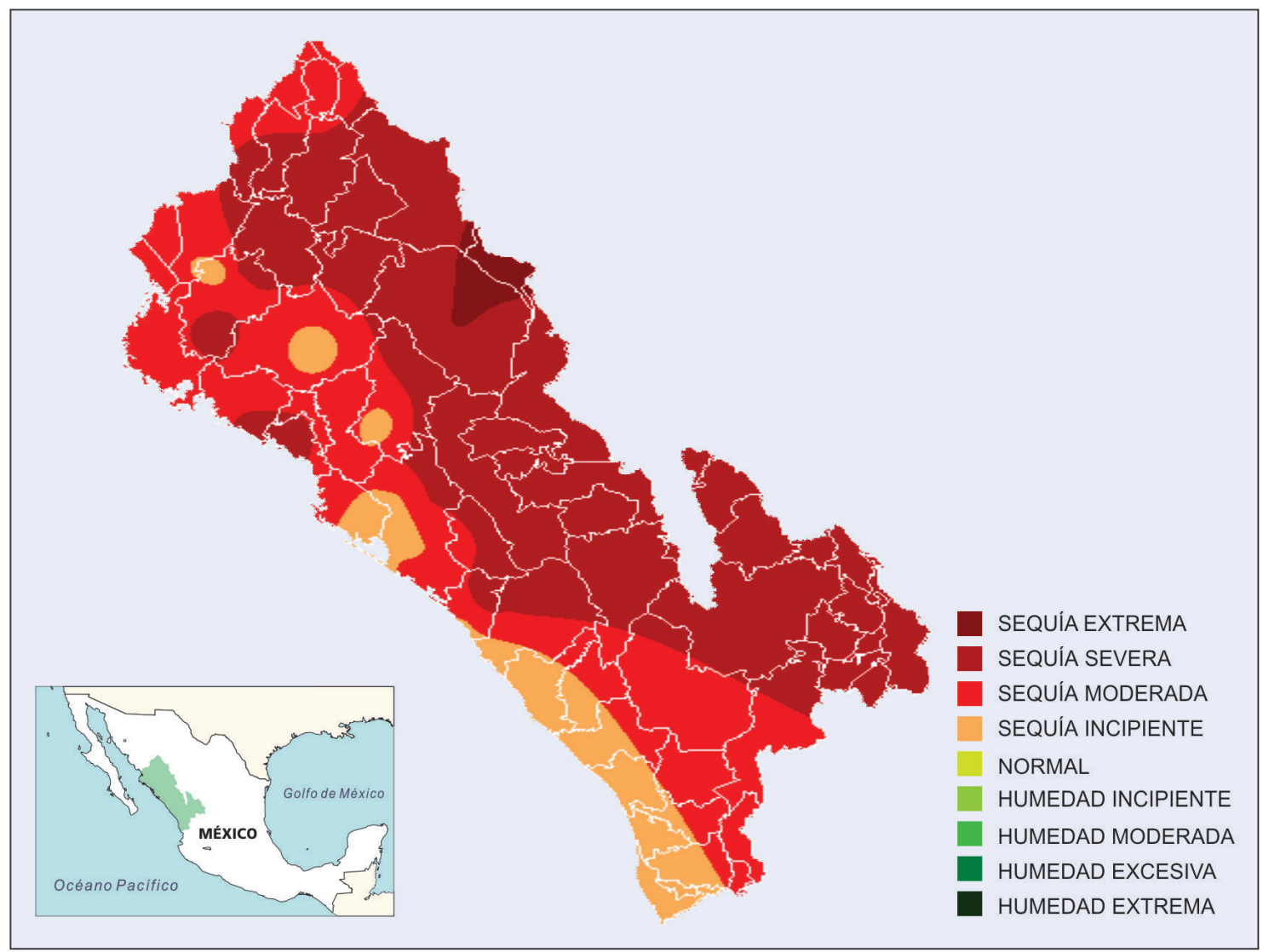

Figura 6. Índice de sequía de Palmer (PDSI) para febrero de 2012 en el área de estudio. Se destacan en rojo las extensas áreas con sequías severas. 
pronosticado para febrero en el mes anterior y el calculado con los datos del mes de febrero. La verificación de la precisión de los pronósticos se presenta en la Tabla 2. Se debe destacar que el coeficiente de Pearson es siempre igual o superior a 0,84 para pronósticos a 1 mes y a 0,60 para pronósticos a 3 meses. Estos coeficientes indican una buena correlación entre el índice de sequía calculado con los datos del mes y el valor pronosticado mediante las redes neuronales. Los errores entre el valor calculado y el pronosticado no superan los límites de la clase de sequía, excepto para la localidad Varejonal y para pronósticos a 2 y 3 meses. Esto significa que si bien hay diferencias numéricas entre ambos valores, el pronóstico corresponde a la misma clase de sequía que el índice calculado para ese mes. Resultados similares fueron obtenidos por Serio et al. (2010) en la evaluación

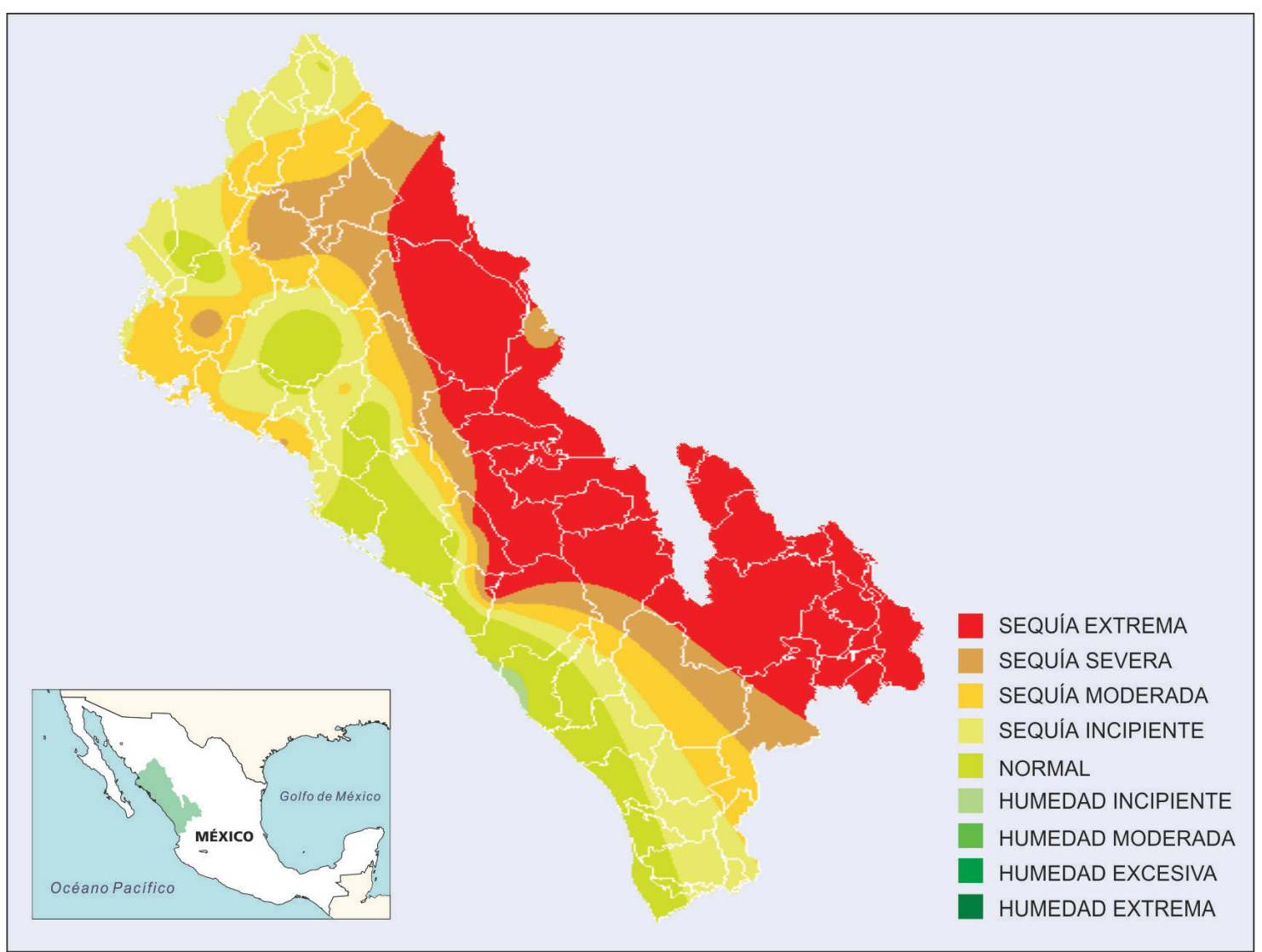

Figura 7. Índice estandarizado de precipitación SPI12 para febrero de 2012 en el área de estudio. Se destacan en rojo las extensas áreas con sequías severas.

Tabla 2. Coeficiente de Pearson y errores absoluto medio (MAE) y cuadrático medio (MRSE) entre valores calculados y pronosticados del PDSI mensual para las localidades de Culiacán. Sanalona y Varejonal. Años 2011, 2012 y 2013 ( $\mathrm{n=27).}$

\begin{tabular}{llccc}
\hline Localidades & & Pronóstico a 1 mes & Pronóstico a 2 meses & Pronóstico a 3 meses \\
\hline Culiacán & Coef.de Pearson & 0,88 & 0,87 & 0,78 \\
& MAE & 0,39 & 0,41 & 0,60 \\
& MRSE & 0,50 & 0,55 & 0,74 \\
Sanalona & Coef.de Pearson & 0,84 & 0,69 & 0,60 \\
& MAE & 0,67 & 0,85 & 1,07 \\
& MRSE & 0,89 & 0,97 & 1,15 \\
& Coef.de Pearson & 0,94 & 0,86 & 0,85 \\
& MAE & 0,43 & 0,71 & 0,78 \\
\hline
\end{tabular}




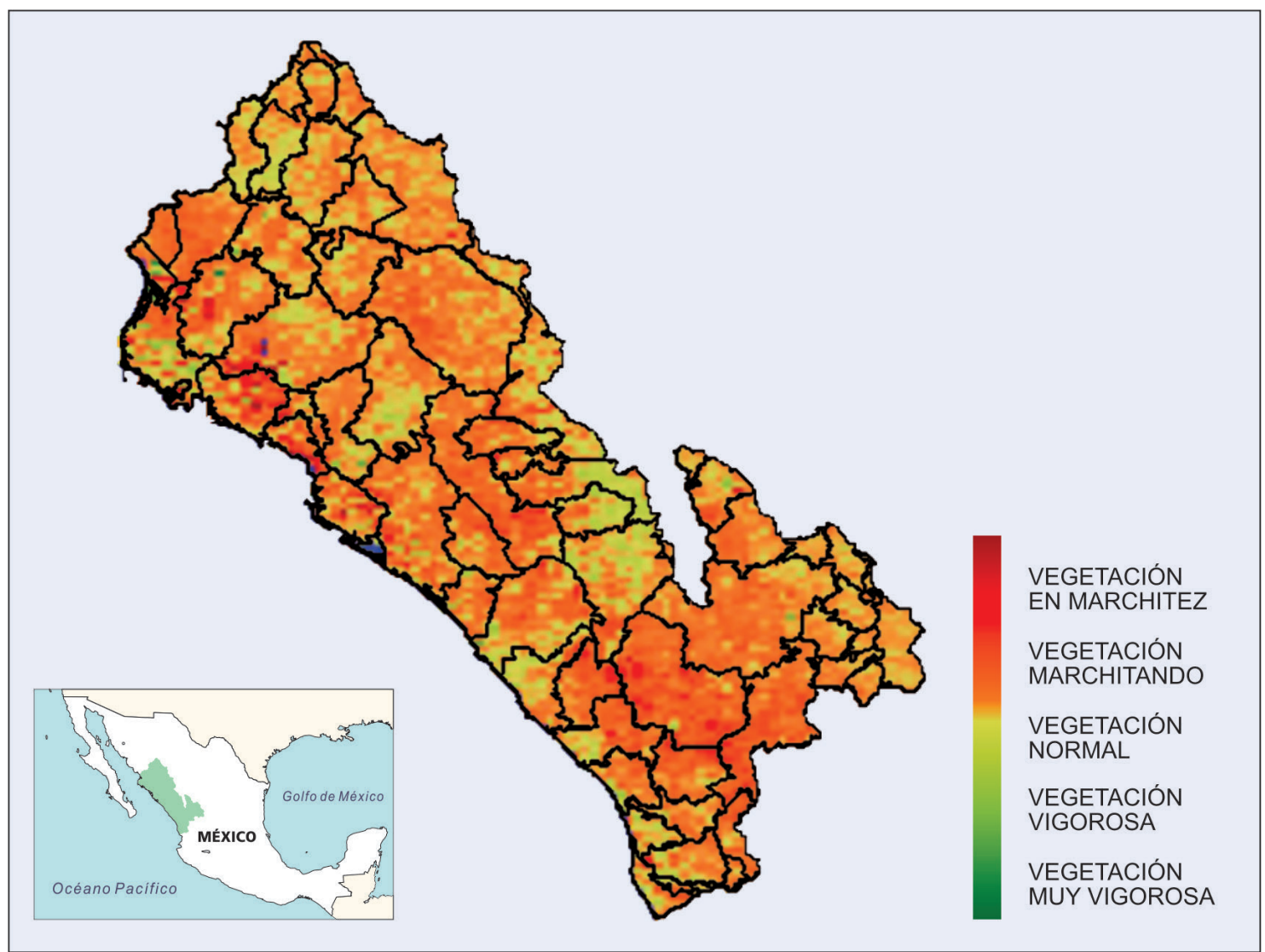

Figura 8. Índice de vegetación (NDVIDif) para febrero de 2012 en el área de estudio.

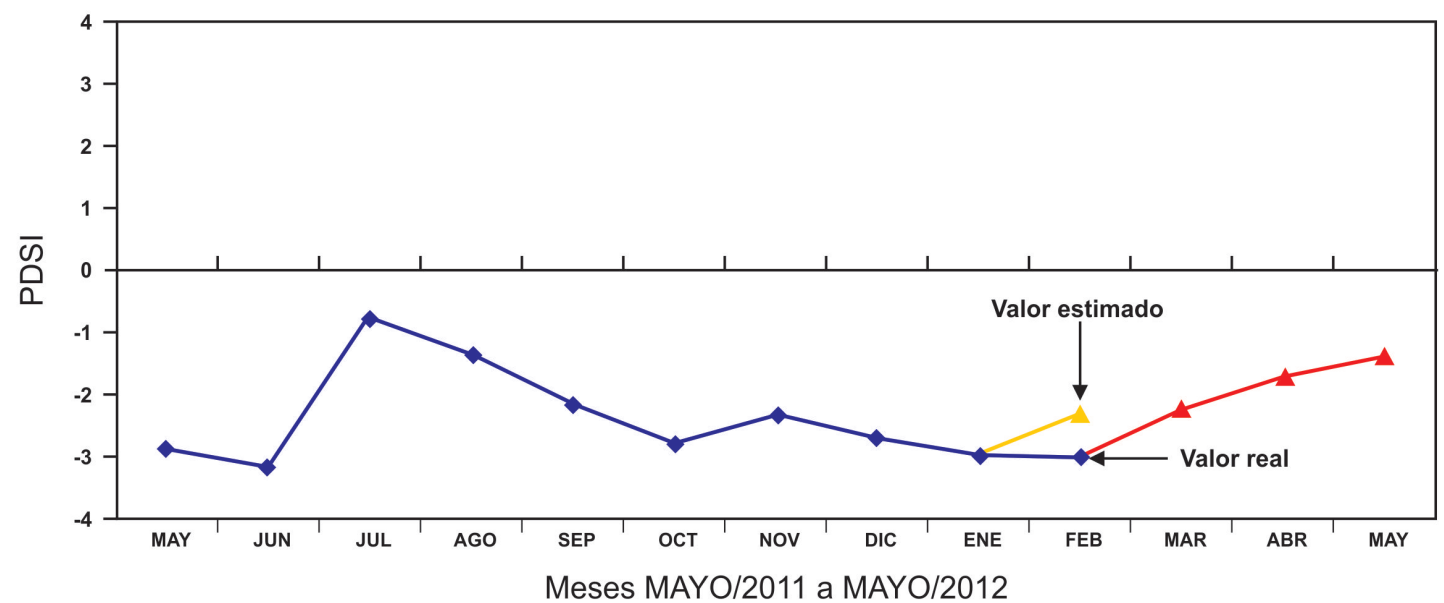

Figura 9.Pronóstico del índice de severidad de sequía de Palmer realizado en febrero para los meses de marzo a mayo de 2012 (en rojo) para la estación El Varejonal. Valor pronosticado (anaranjado) en enero y verificado con el índice de febrero. La diferencia entre ambos valores indica el error del pronóstico.

de la precisión del SPI como indicador de la ocurrencia de sequías.

Las redes neuronales son sistemas dinámicos autoadaptativos; son adaptables, debido a la ca- pacidad de autoajuste de los elementos procesales (neuronas) que componen el sistema, y son dinámicos porque son capaces de estar constantemente cambiando para adaptarse a las nuevas 
condiciones. Estas características son especialmente importantes para el análisis de las condiciones ambientales relacionadas con las sequías. La capacidad de pronosticar condiciones hídricas (ocurrencia o persistencia de sequías o no) mediante el análisis de condiciones próximo pasadas las convierte en una poderosa herramienta de análisis (Shin \& Salas, 2000).

Los pronósticos de las condiciones hídricas pueden hacerse para toda una región o una cuenca en particular mediante el uso de un sistema de información geográfica y un índice de sequía, tal como lo presenta la Figura10 para la cuenca del río Culiacán, realizada en febrero y los pronósticos para los meses de marzo a mayo de 2012. Puede apreciarse una mejora de las condiciones de sequías en la cuenca media y alta desde marzo (sequías severas y moderadas) a mayo (sequias incipientes). La cuenca baja se encontró siempre con sequías incipientes.

En el OCPN se desarrolla una actividad de seguimiento de las condiciones hídricas en toda la región y se publica mensualmente un boletín que contiene los resultados del monitoreo, evaluación y pronóstico de las sequias como los presentados precedentemente (CONAGUA, 2013).

El uso simultáneo de índices meteorológicos e índices de vegetación derivados de información satelital mejora la detección y evaluación de las condiciones hídricas. En India, Bhuiyan et al. (2006) usaron con éxito dichos índices para la identificación de sequías, y encontraron que el índice satelital de vegetación posee mayor inercia que los índices meteorológicos para señalar el comienzo y fin de las sequías. En este estudio, se identifica una relación estrecha entre el PDSI y el NDVIDif, pero este posee una mejor resolución espacial. Dicha relación ha sido utilizada para generar un índice

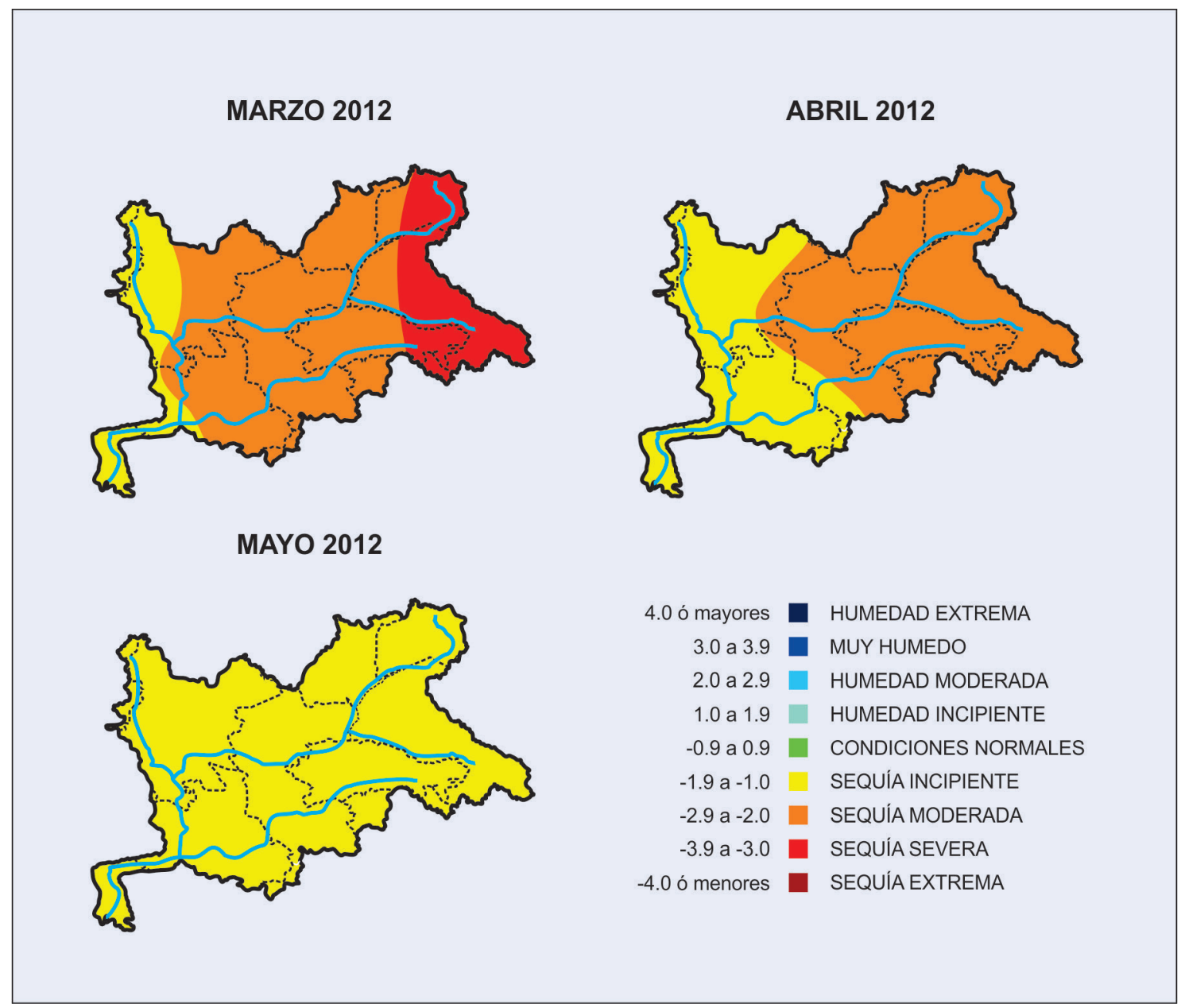

Figura 10.Pronóstico del índice de severidad de sequía de Palmer para la cuenca del río Culiacán (Sinaloa) realizado en febrero y para el período marzo-mayo, 2012. Líneas de puntos indican los límites municipales y líneas azules continuas los principales ríos de la cuenca. 
combinado de sequías con una resolución espacial de $1 \mathrm{~km}$, que facilita la toma de decisiones a nivel local (Brown et al., 2008).

En China se utilizaron series plurianuales del NDVI para identificar la ocurrencia de sequías de larga duración a través de sus efectos en la vegetación (Wei Wanga et al., 2010), cuyos resultados avalan el uso del NDVI como indicador de sequias y son concordantes con los hallados en este trabajo. Asimismo, la utilización de índices derivados del NDVI para el monitoreo de las sequías ha mejorado la precisión de su identificación y evaluación (Peters et al., 2002).

Los pronósticos de las sequías obtenidos a nivel local y regional mediante el uso de redes neuronales concuerdan en precisión con los resultados obtenidos por Shin \& Salas (2000). Sin embargo, el uso generalizado de redes neuronales para análisis multivariado con varios índices de sequía requiere de investigaciones adicionales (Douriet, 2013b).

Aun con la utilización simultánea de índices de sequías, la identificación de los efectos adversos de las sequías es difícil y compleja (Wilhite et al., 2007). Por ello es necesario el desarrollo de sistemas ad hoc que consideren los niveles de vulnerabilidad de los diferentes sectores productivos y de la sociedad, aspectos que han sido considerados en Ravelo (2013).

\section{CONCLUSIONES}

El uso de diferentes índices de sequías permitió un enfoque multifacético de la adversidad climática y su visualización temporal y regional mediante ilustraciones específicas (gráficos y mapas). Por otro lado, se establecieron las bases para el comienzo de actividades en el monitoreo a tiempo real de las condiciones hídricas con énfasis en las sequías, su evaluación y pronóstico a corto y mediano plazo. La disponibilidad actualizada de los resultados del programa SOS permitirá que los sectores públicos y privados tomen decisiones acertadas para el uso eficiente y sostenible de los recursos hídricos. A su vez, los pronósticos de sequía permitirán una administración programada del uso de las reservas hídricas de las presas. La perdurabilidad y el mejoramiento del sistema quedarán asegurados en la medida que los usuarios verifiquen los beneficios que se logran en el manejo integrado del agua, en el cual la detección, evaluación y pronóstico de las sequías poseen un rol relevante.

La gravedad de los efectos adversos generados por las sequías requiere del esfuerzo conjunto de las diferentes esferas de gobierno, las universi- dades, los centros de investigación y la sociedad representada por los diferentes usuarios del agua, así como del conocimiento más profundo del comportamiento del fenómeno.

\section{AGRADECIMIENTOS}

Al personal de OMM/México y de CONAGUA por la provisión de datos y por las valiosas sugerencias y recomendaciones durante el desarrollo del proyecto PREMIA. A Guillermo Marraco, Antonio Mendivil y Victor Ornelas por el invalorable apoyo informático. Alejandro Barbeito preparó las ilustraciones.

\section{BIBLIOGRAFIA}

Alley, W.M., 1984. The Palmer Drought Severity Index: limitations and assumptions. Journal of Climate and Applied Meteorology 23:1100-1109.

Bhuiyan, C.; R.P. Singh and F.N. Kogan, 2006. Monitoring drought dynamics in the Aravalli region (India) using different indices based on ground and remote sensing data. Int. J. Applied Earth Observation and Geoinformation 8:289-302.

Brown, J.F., B.D. Wardlow, T. Tadesse, M.J. Hayes and B.C. Reed, 2008. The Vegetation Drought Response Index (VegDRI): A New Integrated Approach for Monitoring Drought Stress in Vegetation. GIScience \& RemoteSensing 45(1):16-46.

CONAGUA, 2012. Comisión Nacional del Agua/ Secretaria de Medio Ambiente y Recursos Naturales. Organismo de Cuencas del Pacífico Norte. Informes del estado de las presas. <http://www.conagua.gob.mx/OCPN07/ Contenido/ Documentos/PRESASgrpn.pdf> Consultada el 22/03/2013.

CONAGUA, 2013. Comisión Nacional del Agua/ Secretaria de Medio Ambiente y Recursos Naturales. Organismo de Cuencas del Pacífico Norte. Boletines de monitoreo, evaluación y pronóstico de sequias. <http://www.conagua .gob.mx/ocpn/Contenido.aspx?n1=2\&n2=5\&n3 = 11> Consultada el 12/06/2013.

CONAGUA, 2014. Comisión Nacional del Agua/ Secretaria de Medio Ambiente y Recursos Naturales. Programa de Medidas Preventivas y de Mitigación de la Sequía, Consejos de Cuenca Ríos Presidio al San Pedro, Rios Mocorito al Quelite y Fuerte al Sinaloa. $<$ http://www.conagua.gob.mx/pronacose /Contenido. aspx?n1=7\&n2=54> Consultada el 12/01/2014.

CREAN, 2012. Centro de Relevamiento y Evaluación de Recursos Agrícolas y Naturales, CONICET/UNC. Monitoreo de Sequias. <http://crean.org .ar/monitoreo> Consultada el 22/05/2013. 
Douriet, J.C., 2010. Caracterización y evaluación de escenarios por sequia en la cuenca y acuífero del río Culiacán. Tesis de maestria. <www.imta.edu.mx/images/pdf/ tesis_jose\%20carlos\%20douriet\%20cardenas_3.pdf> Consultada el 22/03/2013.

Douriet J.C., 2013a. Pronóstico de Sequías en la Cuenca del río Culiacán, Sinaloa, México. V Convención Cubana de Ciencias de la Tierra (Geociencias 2013), Palacio de Convenciones, La Habana, Cuba. <http://www. cubacienciasdelatierra.com/Memorias2013/MemoriasTrabajos \%20Y\%20Resumenes2013.pdf> Consultada el 2/01/2014.

Douriet, J.C., 2013b. Determinación de escenarios por sequía mediante el análisis multivariado temporal usando redes neuronales: Caso de estudio en el Organismo de Cuenca Pacífico Norte, México. Tesis doctoral. Escuela para Graduados, Fac. C. Agrop., Univ. Nac. de Córdoba.

Eastman, R., 2012. IDRISI Selva. Guide to GIS and Image Processing- ClarkLabs Clark University, Worcester, Massachusetts, U.S.A. En CD.

EIRD/ONU, 2009. Estrategia internacional para la reducción de desastres. Organización de las Naciones Unidas. <www.eird.org/eng/revista/no-16-2009/art18.html> Consultada el 20/10/2013.

Hayes, M. J.; M.D. Svoboda, D.A. Wilhite and O.V. Vanyarkho, 1999. Monitoring the 1996 drought using the standardized precipitation index. Bull. American Meteorology Society 80:420-438

IWMI, 2012. Datos de evapotranspiración potencial. International Water Management Institute. <www.iwmi.cgiar. org/WAtlas/AtlasQuery.htm> Consultada el 12/03/2013.

Justice, C.O. and J.R.G. Townshend, 2002. Special issue on the Moderate Resolution Imaging Spectroradiometer (MODIS): A new generation of land surface monitoring. Remote Sens. Environ. 83: 1-2.

Kogan, F.N. (1997) Global drought watch from space. Bulletin of the American Meteorological Society 78: 621-636.

Liu, W.T. and F.N. Kogan, 1996. Monitoring regional drought using the vegetation condition index, International Journal of Remote Sensing 17(14):2761-2782.

McKee, T.B.; N.J. Doesken and J. Kleist, 1993. The relationship of drought frequency and duration to time scales. Preprints, Eighth Conference on Applied Climatology, Anaheim, California, Amer. Meteor. Soc., pp. 179-184.

Mishra, A.K. and V.R. Desai, 2006. Drought forecasting using feed-forward recursive neural network. Ecol. Modelling 198:127-138

Morales, A.; J. Olcina y A. Rico, 2000. Diferentes percepciones de las sequías en España: adaptación, catastrofismo e intentos de corrección. Investigaciones Geográficas $n^{\circ}$ 23. Instituto Universitario de Geografía.
Universidad de Alicante.

NOAA, 2012. National Weather Service, Climate Prediction Center. Drought monitoring. <http://www.cpc.ncep. noaa.gov/products/analysis_monitoring/cdus /palmer_ drought/wpdanote.shtml> Consultada el 2/11/2013.

Palmer, W.C., 1965. Meteorological drought. Documento de Investigación N 45, Oficina Meteorológica del Departamento de Comercio, Washington, D.C.

Peters, A.J., E.A. Walter-Shea, I. JI, A. Viña, M. Hayes and M.D. Svoboda, 2002. Drought Monitoring with NDVI-Based Standardized Vegetation Index. Photogrammetric Engineering \& Remote Sensing 68(1):71-75.

Planchuelo, A.M. and A.C. Ravelo, 1985. Drought/Crop Condition Assessment Methods for Northeast Brazil, Mexico, Paraguay and Uruguay. Edit. Univ. Missouri Press. Missouri, U.S.A. 128 pp.

Ravelo, A.C., 1973. Método AGG de estimación de las temperaturas medias mensuales. RIA, INTA, Series 3, Vol. X, No. 5.

Ravelo, A.C., 1980. Drought/Food production problems in Haiti: A case study for 1978. Tech. Report to USAID/ OFDA, $57 \mathrm{pp}$.

Ravelo, A.C., 2000. Caracterización Agroclimática de las sequías extremas en la región pampeana argentina. Rev. Facultad Agronomía 20(2): 187-192.

Ravelo, A.C., 2012. Informes Finales del Proyecto PREMIA. OMM - CONAGUA. <https://sites.google.com/a/wmo. int $/$ mex/system/app/pages/search?scope= searchsite\&q=ravelo $>$ Consultada el 22/08/2013.

Ravelo, A.C. y M. Herrero, 1999. Indice de sequía de Palmer. Software académico. Registrado en Direccion Nac. Derechos de Autor No. 30666/99. En CD.

Ravelo A.C., R. Zanvettor y W. Da Porta, 2001. Desarrollo de un sistema para la detección, seguimiento y evaluación de las sequías agrícolas en Argentina. Revista Argentina de Agrometeorología 1(2): 27-34.

Sanz Ramos, R.; J.C. Douriet Cardenas, A. Ravelo y V.J. Ornelas Sánchez, 2012. Monitoreo de la sequía 20112012 en la RH10-OCPN. XXII Congreso Nacional de Hidráulica. <http://www.revistatlaloc.org.mx/amh_congreso /articulos/CambioClimaticoyEventosExtremos/02 1Art_rsr1.pdf> Consultada el 22/11/2013.

Serio, L; P. Martín y G. Murphy, 2010. Evaluación de una metodología de pronóstico estadístico para la condición hídrica del suelo en la región pampeana argentina. Agriscientia 27(1): 11-17

Shin H-S and J.D. Salas, 2000. Regional Drought Analysis Based on Neural Networks. J. Hydrologic Eng. 5(2):145155.

Tucker, C.J., 1979. Red and Photographic Infrared Linear Combinations for Monitoring Vegetation, Remote Sensing of Environment, 8(2),127-150. 
USNDPC, 2000. Preparing for drought in the 21st century : United States National Drought Policy Commission Report, Dept. of Agriculture. Office of Communications. 60 pp. <http://govinfo.library.unt.edu/drought/finalreport/ fullreport/pdf/reportfull.pdf> Consultada el 12/11/2012.

Wei Wanga; Wen-jie Wang, Jun-sheng Li, Hao Wu, Chao $\mathrm{Xu}$ and Tan Liu, 2010. The Impact of Sustained Drought on Vegetation Ecosystem in Southwest China Based on Remote Sensing. Procedia Environmental Sciences 2: 1679-1691.

White, D.H. and J.J. Walcott, 2009, 'The role of seasonal indices in monitoring and assessing agricultural and other droughts: a review'. Crop and Pasture Science 60 (7): 599-616.

Wilhite, D.A.; M.D. Svoboda and M.J. Hayes, 2007. Understanding the complex impacts of drought: A key to enhancing drought mitigation and preparedness. Water Res. Man. 21:763-774

Zanvettor, R.E and A.C. Ravelo, 2000. Using the SPI to monitor the 1999-2000 drought in Northeastern Argentina. Drought Network News 12(3): 3-4. 